
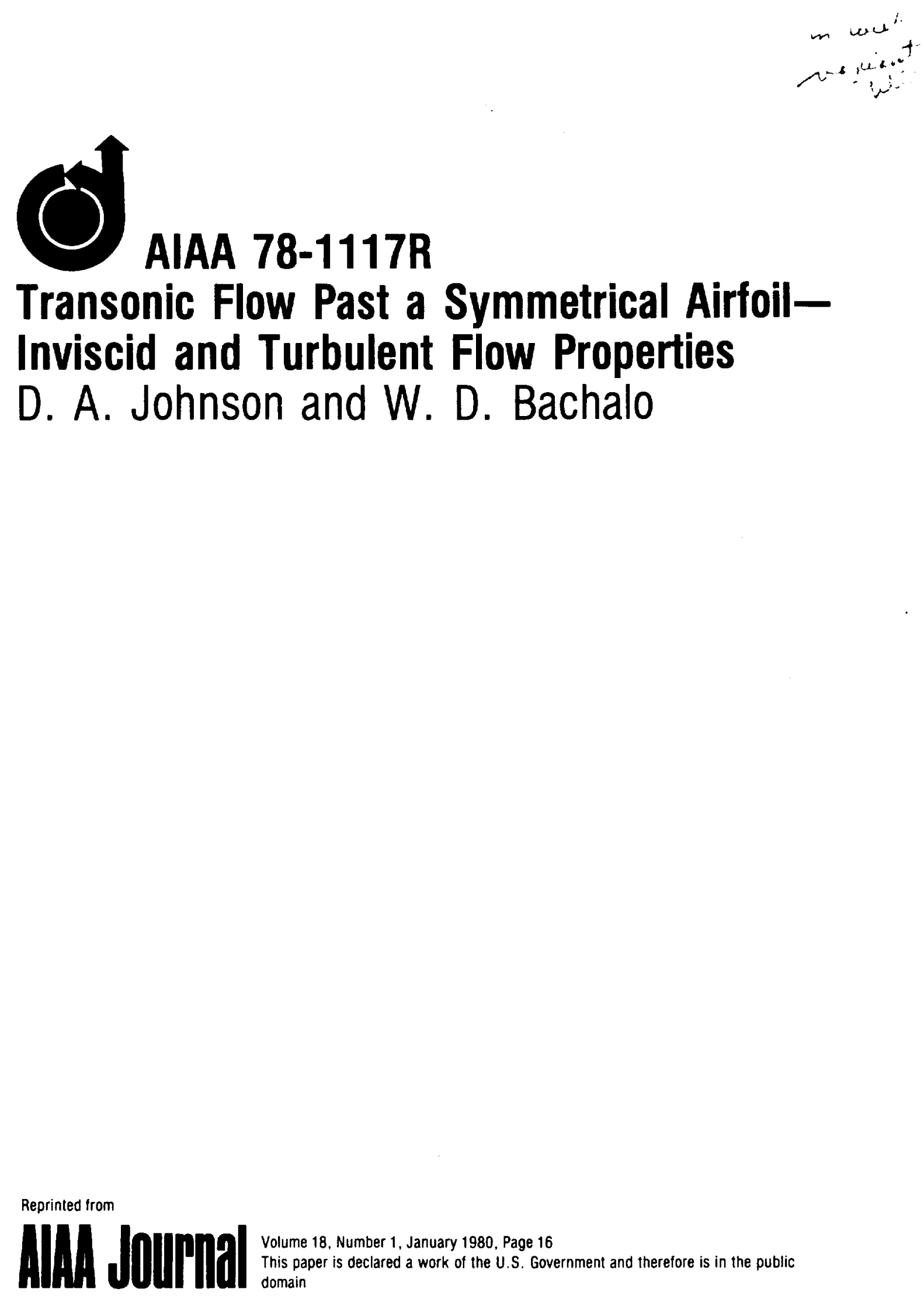


\title{
Transonic Flow Past a Symmetrical Airfoil-Inviscid and Turbulent Flow Properties
}

\author{
D. A. Johnson* and W. D. Bachalo ${ }^{*}$ \\ NASA Ames Research Center, Moffett Field, Calif.
}

\begin{abstract}
Flowfield measurements are presented for a symmetrical NACA $64 A 010$ airfoil section at Iransonic conditions. Measurements were oblained for three angles of attack with the freestream Mach number fixed at 0.8 . The cases studied included a weak shock-wave/boundary-layer interaction, an interaction of medium strength with mild separation, and an interaction of sufficient strength to produce a shock-induced stall situation. Two nonintrusive optical techniques, laser velocimetry and holographic interferometry, were used to characterize the flows. The results include Mach number contours and flow angle distributions in the inviscid flow regions, and turbulent flow properties, including the turbulent Reynolds stresses, of the upper surface viscous layers, and of the near-wake. The turbulent flow measurements reveal that the turbulence fluctuations attain equilibrium with the local mean flow much faster than previously expected.
\end{abstract}

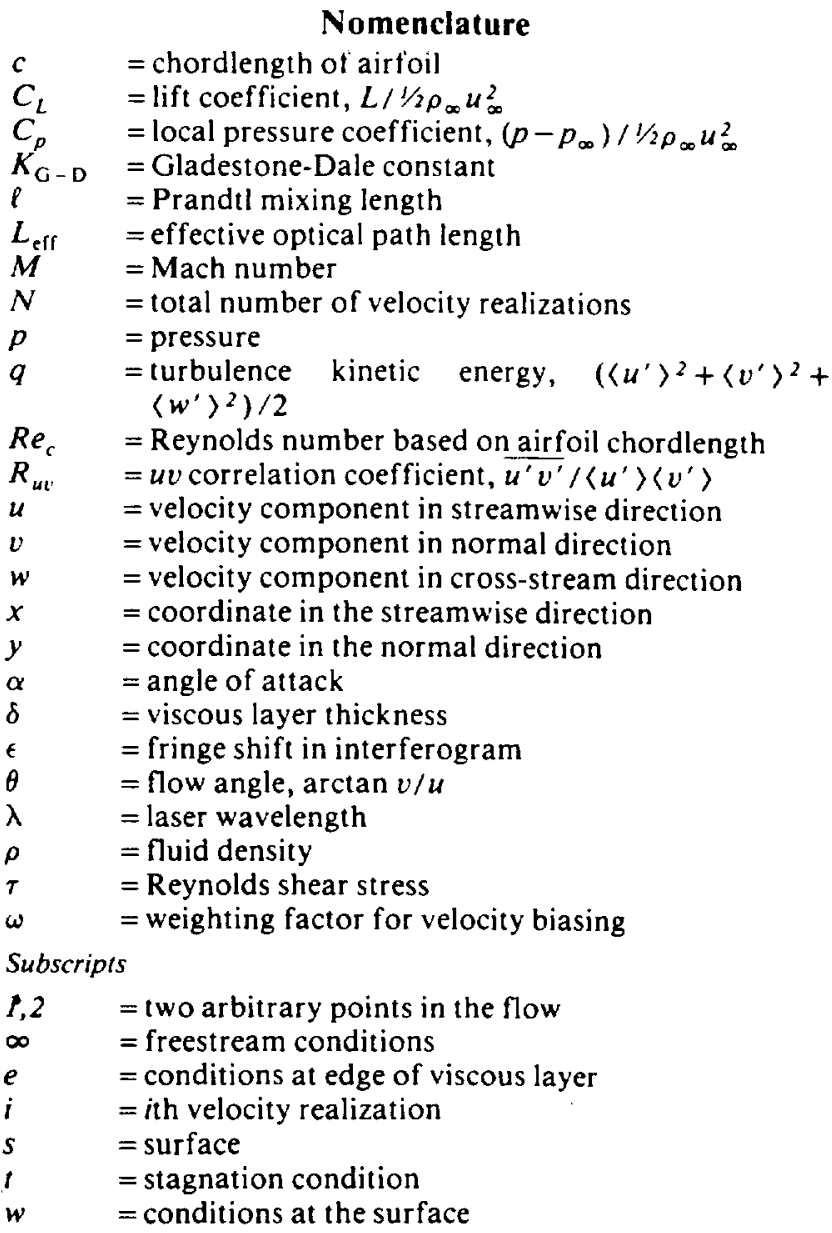

Presented as Paper 78-1117 at the AlAA 11th Fluid and Plasma Dynamics Conference, Seattle, Washington, July 10-12, 1978; sub. mitted Jan. 1, 1979; revision received May 22, 1979. This paper is declared a work of the U.S. Government and therefore is in the public domain. Reprints of this article may be ordered from AIAA Special Publications, 1290 Avenue of the Americas, New York, N.Y. 10019 Order by Article No. at top of page. Member price $\$ 2.00$ each, nonmember, $\$ 3.00$ each. Remittance must accompany order.

Index categories: Aerodynamics; Transonic Flow; Boundary Layers and Convection Heat Transfer-Turbulent.

- Research Scientist. Member AlAA.

†Staff Scientist, Spectron Development Lab. Member AlAA.
Superscripts

()$^{\prime}$ = fluctuating quantity

( ) = time-averaged quantity

$\left\langle{ }^{\prime}\right\rangle \quad=$ rms value of quantity

\section{Introduction}

A LTHOUGH considerable advances have been made toward the numerical prediction of the transonic flow past two-dimensional airfoil sections (e.g., Refs. 1-5), these numerical methods currently have a limited range of applicability, either because they ignore viscous effects altogether or because they inadequately predict the viscous effect as the shock wave strengthens on the airfoil's upper surface. Accurate predictions are especially difficult to obtain once shock-induced separation occurs. This inability to account accurately for viscous effects is a direct consequence of the deficiencies in the models employed for the turbulent Reynolds stresses (e.g., Ref. 4). To assess truly the ability of these methods to describe the flow behavior and to provide insight into how to improve on these methods, quantitative measurements of the external flow and, especially, of the turbulent transport properties, are needed. In transonic testing, flowfield data are also needed if account is to be taken of wall interference effects which can alter significantly the pressure distributions from those that would be measured under free-air conditions.

The objective of the present study was to obtain detailed descriptions of both the inviscid and viscous flow regions including the turbulence transport properties about a geometrically simple, symmetrical airfoil section (NACA $64 \mathrm{A010}$ ) for several different test conditions. The conditions selected for study were: a weak shock-wave/boundary-layer interaction for which inviscid methods should be adequate, an interaction of medium strength containing some separation, and a strong interaction with massive separation as occurs at stall. The severity of the interaction was established by varying angle of attack at a fixed freestream Mach number of 0.8 . To describe the flow about the airfoil section quantitatively, two nonintrusive optical methods were employed. A laser velocimeter technique was used to provide detailed localized information of the streamwise and normal velocity components (mean and fluctuating). Holographic in. terferometry was used to obtain the density fields about the airfoil section from which Mach number contours were determined. For completeness of the experiment, surface pressure distributions, wake pressure, oil flow visualizations, and conventional Schlieren and shadowgraphs were also obtained. 


\section{Experimental Technique}

The experiments were conducted in the Ames 2- by 2-Foot Transonic Wind Tunnel which is a closed-return, variabledensity tunnel with $21 \%$ open porous-slotted upper and lower walls for transonic testing. A 6 -in. chord 64A010 airfoil which spanned the entire test section was selected for the tests. Tunnel conditions were fixed at a freestream Mach number of 0.8 and a chord Reynolds number of $2 \times 10^{6}$. To insure that the boundary layer was turbulent at the foot of the shock wave for all test conditions, transition strips were affixed to the airfoil section at the $17 \%$ chord station on the upper and lower surfaces. The 1.25 -mm-wide strips consisted of 0.13 $\mathrm{mm}$ nominal diameter glass bends, selected in accordance with the recommendations of Ref. 6 . Transition effectiveness was verified by the sublimination technique.

\section{Laser Velocimeter}

The laser velocimeter was a dual-color system utilizing the $4880 \AA$ and $5145 \AA$ lines of an argon-ion laser; one spectral line to measure the streamwise velocity component, the other to measure the vertical velocity component. Bragg-cell frequency shifting, necessary for probing highly turbulent and separated flow regions, was incorporated in both spectral lines. The frequency offsets also facilitated the direct measurement of the vertical velocity component (i.e., \pm 45 deg beam orientations to resolve the vertical velocity were unnecessary). The effective sensing volume was approximately a cylinder, $200 \mu \mathrm{m}$ in diameter and $3 \mathrm{~mm}$ long, whose axis was aligned with the cross-stream direction.

Signal processing was accomplished with single-particle burst counters and the individual realizations from the two channels were recorded simultaneously with a digital com. puter. This allowed the velocity correlation $u^{\prime} v^{\prime}$ to be obtained in a straightforward manner by multiplying and averaging, rather than by the less accurate method of subtracting the variances of the two signals obtained from \pm 45 deg beam orientations. In addition, it facilitated the weighting of the data for the velocity biasing which is believed to occur when the particle concentration is low. This biasing is attributed to particle arrival rate dependency on instantaneous velocity. In this paper, the data presented were adjusted using the two-dimensional weighting factor suggested in Ref. 7. The mean velocities, turbulence intensities, and velocity correlations $\overline{u^{\prime} v} v^{\prime}$ were calculated as follow's:

$$
\begin{aligned}
\bar{u} & =\sum_{i=1}^{N} \omega_{i} u_{i} / \sum_{i=1}^{N} \omega_{1} \\
\left\langle u^{\prime}\right\rangle & =\left[\left(\sum_{i=1}^{N} \omega_{i} u_{i}^{2} / \sum_{i=1}^{N} \omega_{i}\right)-\bar{u}^{2}\right]^{\prime} \\
\overline{u^{\prime} v^{\prime}} & =\left(\sum_{i=1}^{N} \omega_{1} u_{i} v_{1} / \sum_{i=1}^{N} \omega_{i}\right)-\overline{u v}
\end{aligned}
$$

with the two-dimensional weighting factor given by

$$
\omega_{i}=1 / \sqrt{u_{i}^{2}+v_{i}^{2}}
$$

The equations for $\bar{v}$ and $\left\langle v^{\prime}\right\rangle$ are identical to Eqs. (1) and (2), except $v_{i}$ is used in place of $u_{i}$. When no correction for velocity biasing is applied, $\omega_{i}$ is taken to be unity; the data were also reduced in this manner. A comparison of the two sets of results showed that the same overall conclusions regarding the flow behavior would be drawn using either approach.

Naturally occurring particles in the tunnel were used for light scattering. In this facility, lubrication oil within the drive system vaporizes and then later condenses in the tunnel circuit to provide a generous supply of scattering centers. Previous measurements ${ }^{B}$ across a normal shock have shown that these particles are small enough in size (estimated to be $1 \mu \mathrm{m}$ ) to give very good response to a step change in velocity at sonic speeds. At each point in the flow, at least several thousand velocity realizations were used to calculate the flow properties given in Eqs. (1-3). Measured velocities at the edge of the viscous layers were generally repeatable to better than $1 \%$ and agreed well with edge velocities inferred from the measured surface pressures. The primary cause for these variations was changes in the tunnel operating temperature. Under constant test conditions, the inviscid velocities repeated to within $0.25 \%$. In the viscous layers, mean velocities were repeatable to better than $2 \%$ of the edge velocity-including the extremely turbulent separated regions.

\section{Holographic Interferometer}

The holography system was designed to utilize the existing tunnel Schlieren mirrors. The setup is based on the arrangement suggested in Ref. 9 for converting large wind tunnel Schlieren systems to holographic visualization systems. In the present investigation, the dual-plate method ${ }^{10}$ was used wherein an exposure is made on a photographic plate under no-flow conditions and subsequent plates are made at the test conditions. After processing, the no-flow plate and one of the plates taken at test conditions are positioned in a reconstruction plate holder, illuminated with the reference beam and aligned for infinite-fringe interferograms. Vibrations in the system are relatively insignificant since the pulse duration of the ruby laser is extremely short $(=20 \mathrm{~ns})$ and motion between exposures can be eradicated in the reconstruction process.

To reduce the infinite-fringe interferograms to density contours, two-dimensional flow was assumed in the relationship

$$
\rho_{2}-\rho_{J}=\frac{\epsilon}{L_{\mathrm{eff}}} \frac{\lambda}{K_{\mathrm{G}-\mathrm{D}}}
$$

where $\rho_{1}$ and $\rho_{2}$ are the densities at two locations, $\epsilon$ is the fringe shift between the locations, $L_{\text {eff }}$ is the effective optical path length taking into account the sidewall boundary layers, $\lambda$ the laser wavelength, and $K_{\mathrm{G}-\mathrm{D}}$ the Gladestone-Dale constant. Since the correction in optical path length due to the sidewall boundary layers was only $2 \%$, the geometrical width of the tunnel could have been used without any significant loss in accuracy. With the interferometer aligned in the infinite-fringe mode, each fringe corresponds to a constant density contour. Once the density at one point in the flow is established, the remaining contours can be determined from Eq. (5).

Due to the relatively large span of the tunnel, the sensitivity of the system to density changes is quite good for twodimensional testing. At $M_{\infty}=0.8$, for example, one fringe shift corresponds to only about a $0.5 \%$ change in density and a corresponding change in Mach number of about $1 \%$. This and the high aspect ratio (4) of the airfoil tested, which produced good two-dimensionality in the flow, contributed to the quality of data obtainable with this optical method.

\section{Results and Discussion}

Shown in Fig. 1 are the lifting characteristics $\left(C_{L}\right.$ vs $\left.\alpha\right)$ of the $64 \mathrm{~A} 010$ airfoil section for $M_{\infty}=0.8$ as obtained from integration of measured surface pressure distributions. Two sets of data are shown in Fig. 1: those obtained in the present investigation and those from a previous study "in the same facility. The slightly higher lift coefficients observed in the present study are believed to be a result of transition strip placement. In the study of Ref. 11, the transition strips were placed nearer to the leading edge (6.1\% chordwise station). This would produce a thicker turbulent boundary layer at the foot of the shock wave and, hence, a reduction in lift. Other $64 \mathrm{~A} 010$ data, such as those of Stivers, 12 are not included, 


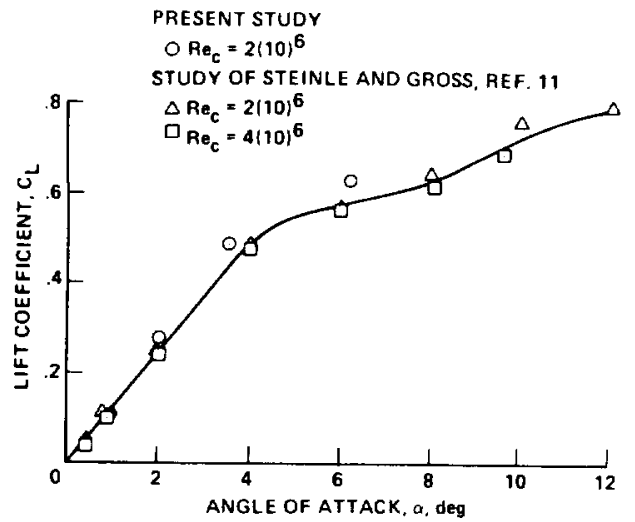

Fig. 1 Lifting characleristics of $64 \mathrm{A010}$ airfoil section for $M_{\infty}=0.8$.

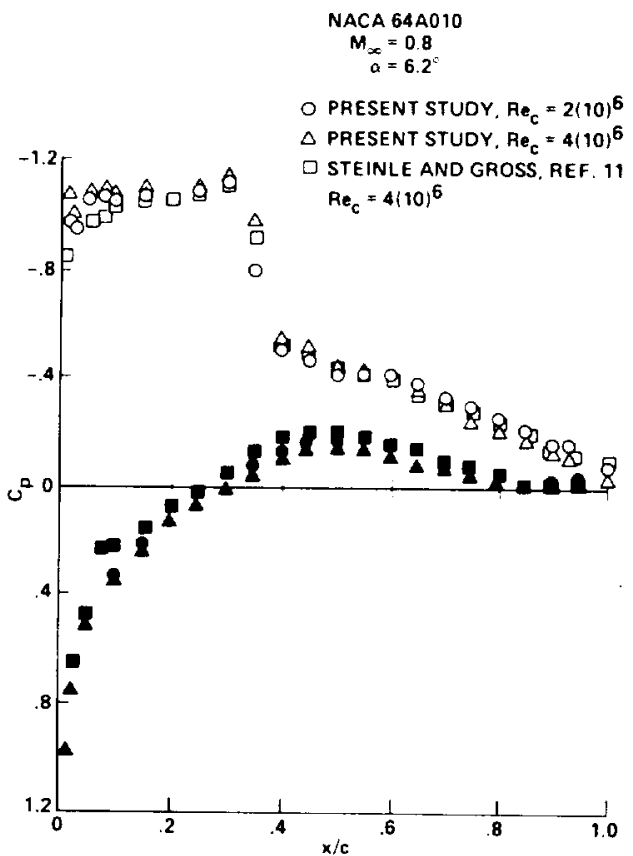

Fig. 2 Reynolds number effects in present study.

since the intent of Fig. 1 is only to show the performance of this airfoil in this particular facility with boundary-layer transition imposed. At the subject Reynolds numbers, substantially different results would have been obtained if natural transition had been employed, as in Ref. 12. It is important to note from Fig. 1 the sudden decrease in the lift curve slope at an angle of attack of about $4 \mathrm{deg}$ which strongly suggests the occurrence of a shock-stall situation. The three angles of attack concentrated on in the present study were set angles of $0,3.5$, and $6.2 \mathrm{deg}$. As is apparent from Fig. 1, the two lifting cases studied occur just prior to and after the sudden decrease in the lift curve slope.

As seen in Fig. 1, the variation in $C_{L}$ due to Reynolds number was observed to be quite small in the study of Ref. 11 . To verify this, surface pressures were obtained for $\alpha=6.2 \mathrm{deg}$ and $R e_{c}=4 \times 10^{6}$ in the present study. These data are compared to data obtained at $R e_{c}=2 \times 10^{6}$ in Fig. 2. Included in Fig. 2 are results from Ref. 11 for $R e_{c}=4 \times 10^{6}$, which show the effect of transition strip placement on the surface pressure distribution. It is evident that the surface pressure distributions for the two Reynolds numbers differ only slightly. The insensitivity to Reynolds number in this study should not be construed to imply that the data would compare well with full-scale Reynolds number results. In the present study, and in that of Ref. 11, the transition strip placement had the effect of nullifying the effects of natural boundary.

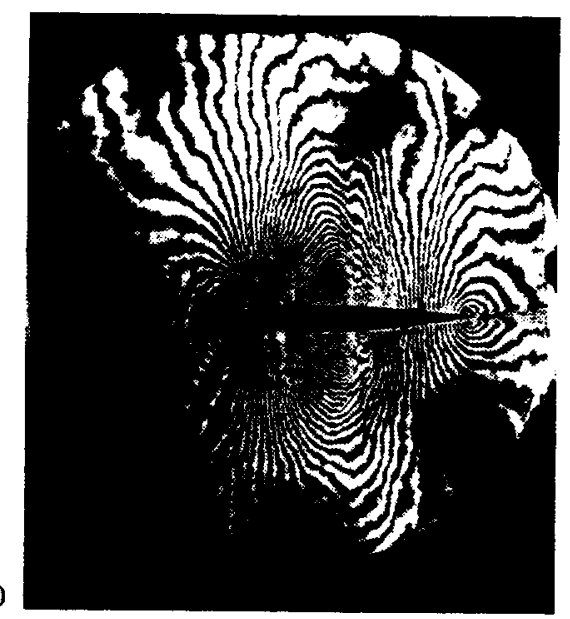

b)
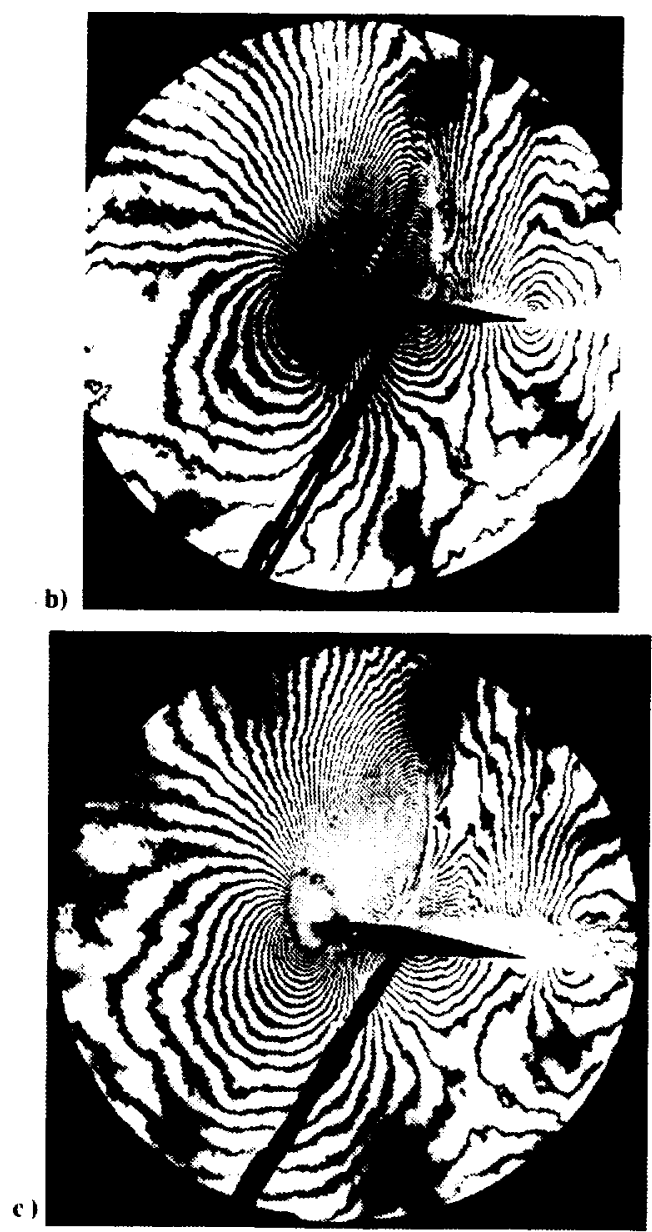

Fig. 3 Infinile-fringe inlerferograms. a) $\alpha=0$ deg; b) $\alpha=3.5$ deg; c) $\alpha=6.2$ deg.

layer transition, thereby reducing the Reynolds number sensitivity. Obviously, the boundary-layer thicknesses relative to the chord of the airfoil in this experiment were much greater than for a flight situation where natural transition occurs. However, the objective of the study was not to obtain data valid for flight conditions but rather to obrain data that could impove our understanding of the basic flow phenomena and, consequently, our predictive capabilities. From this point of view, it is highly desirable not to have the flow be sensitive to the complex transition phenomena.

Infinite-fringe interferograms obtained with the holographic interferometer for the three angles of attack of interest are presented in Fig. 3. As was discussed in the previous section, each fringe corresponds to a line of constant 


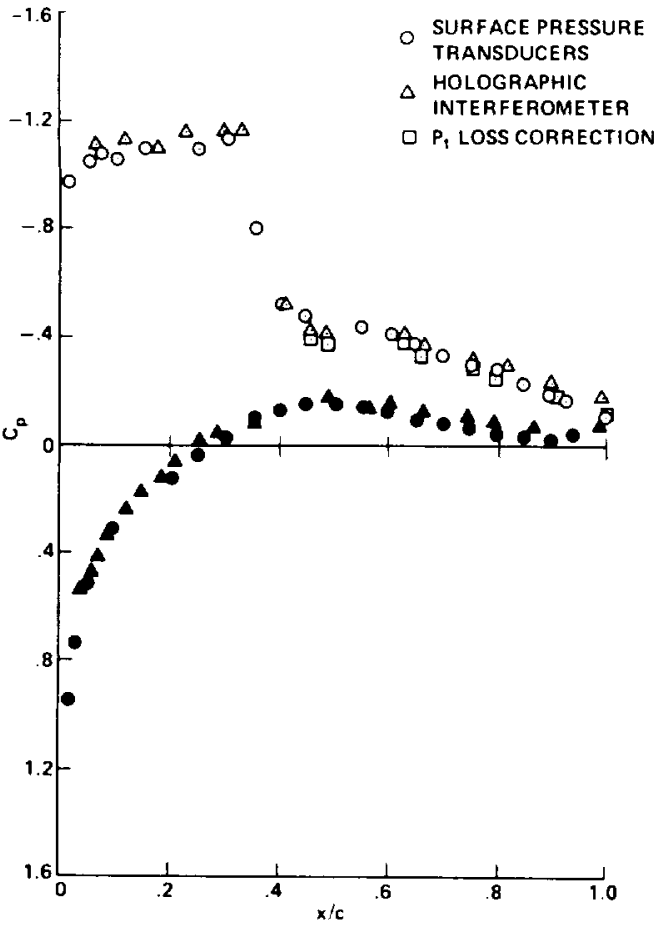

Fig. 4 Surface pressure comparison between directly measured pressures and pressures inferred from interferograms, $\alpha=6.2 \mathrm{deg}$.

density. In the interferograms, the shock wave on the wing's upper surface (upper and lower for the $0 \mathrm{deg}$ case), the turbulent wake and the turbulent boundary layer downstream of the shock, as well as the stagnation region near the leading edge are readily apparent. The major contrast for the three angles of attack is the thickness of the boundary layer on the airfoil's upper surface. For the two lifting cases $(\alpha=3.5$ and $6.2 \mathrm{deg}$ ), the boundary-layer thickness on the upper surface at the trailing edge is, respectively, 2 and 6 times that for the 0 deg case. Also apparent is the very dominant forward curvature in the shock wave near the airfoil for $\alpha=6.2 \mathrm{deg}$, caused by the rapid thickening of the turbulent boundary layer. In the $3.5 \mathrm{deg}$ case, oil flow visualization confirmed that the boundary layer separates at the foot of the shock wave (this was not the case for $\alpha=0 \mathrm{deg}$ ); however, the shock curvature is very much subdued as compared to the $6.2 \mathrm{deg}$ case. Bifurcation (two shock waves) was not observed in any of the cases studied.

Although the interferograms are of value from a nowvisualization point of view, their greatest contribution comes from the quantitative density information they can provide. In principle, if the fluid density can be established at one point in the flow, the density can then be determined at any other point in the flow from Eq (5), provided the flow is twodimensional. The validity of Eq. (5) in the interpretation of these interferograms was established by comparing surface pressure inferred from the interferograms with the directly measured centerline surface pressures. The inferred surface pressures were obtained by assuming isentropic flow in the inviscid region and constant pressure across the viscous layer. The laser velocimeter was used to establish the required density at one point in the flow. In Fig. 4, this comparison is shown for $\alpha=6.2 \mathrm{deg}$. In the $6.2 \mathrm{deg}$ case, the total pressure loss across the shock wave was not neglibile. A correction for this loss in total pressure as determined from the maximum Mach number immediately upstream of the shock wave and the shock-wave inclination is included in Fig. 4. This correction produced better agreement at the trailing edge.

\section{Inviscid Flow Properties}

Under the conditions of isentropic flow, lines of constant density are also lines of constant Mach number. In Fig. 5,
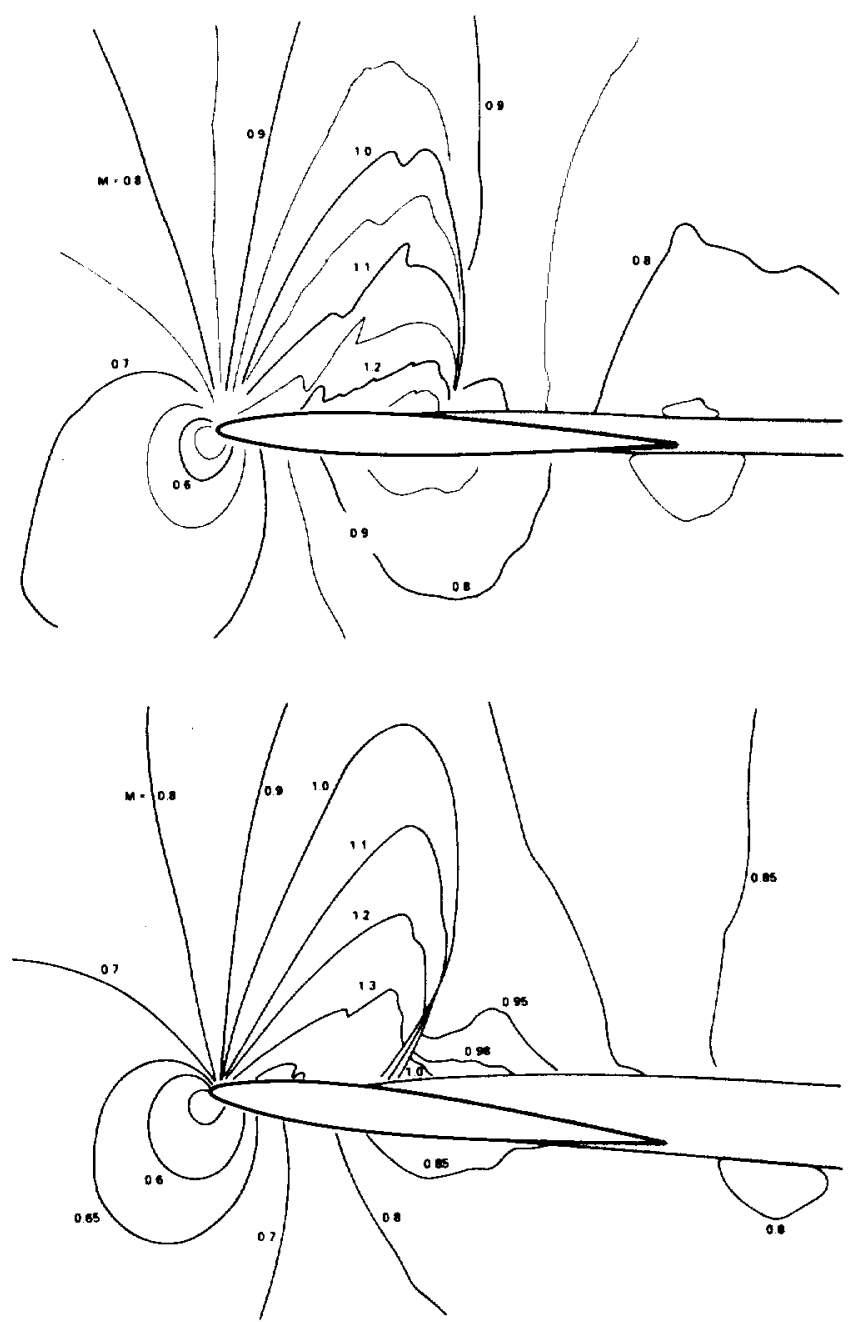

Fig. 5 Mach number contours obtained from interferograms. a) $\alpha=3.5 \mathrm{deg}$; b) $\alpha=6.2 \mathrm{deg}$.

Mach number contour plots obtained from the interferograms under this assumption are presented for the two lifting cases. Although there are total pressure losses across the shock waves, the effect in computing a Mach number is slight. For instance, for the most severe case, $\alpha=6.2 \mathrm{deg}$, the maximum error in Mach number caused by neglecting total pressure losses is only $\Delta M=0.01$.

As seen from Fig. 5, only at the highest angle of attack was a very definite local region of supersonic flow observed downstream of the shock wave. Downstream of this sonic region the flow slowly decelerates. For all three conditions, the shock waves were of the weak solution.

An inviscid flow property not available from interferometry is local flow angle $\theta=\tan ^{-1}(v / u)$. For the three test conditions, horizontal scans were made with the laser velocimeter at $\Delta y / c=0.083$ intervals above the airfoil. The vertical distance was referenced from the chordline at the $25 \%$ chordwise station, which was the point of rotation of the model. Shown in Fig. 6 are near-and far-field results for $\alpha=0$ deg. Included are the flow angles predicted by the method of Ref. 3 using free-air boundary conditions. For the near field $(y / c=0.167)$, the data show a larger flow angle change than that predicted by theory. The reason for the slight differences in the near field may be due to the small disturbance approximations used in the method of Ref. 3. If the particles were unable to track the flow, too small a change in flow angle would be the expected result. In the far field, the experimental data show larger angles in an absolute sense above the trailing edge than above the forward portion of the airfoil. This characteristic was even more pronounced for the lifting 


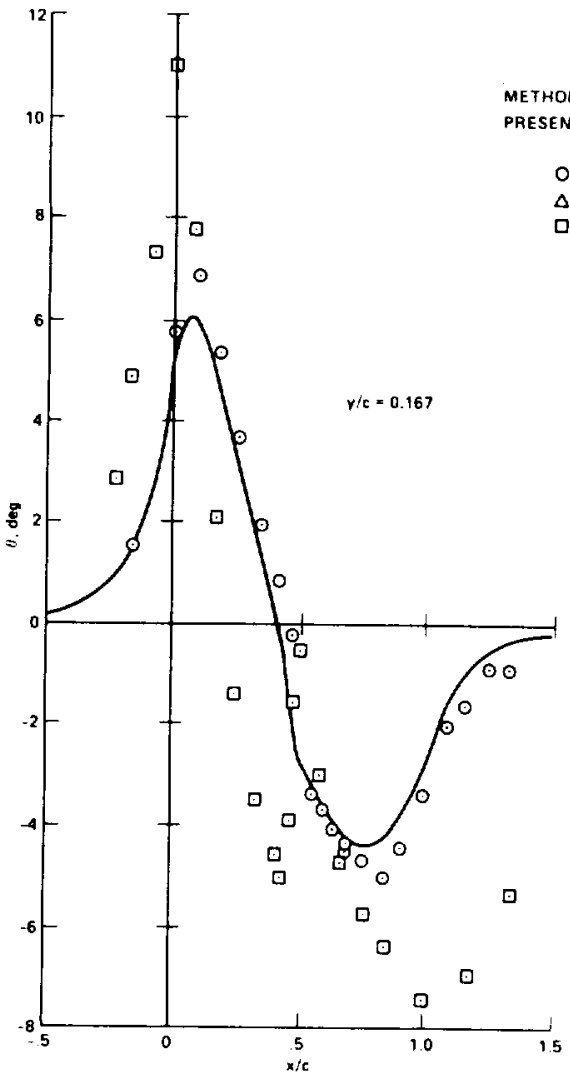

cases, as seen in Fig. 6. Inviscid theory does not predict this trend even when an attempt is made to model the tunnel walls $a^{r}$ is possible with the method of Ref. 3. Surprisingly, the measured far-field flow angles aft of midchord were not measurably different for the two lifting cases. To what degree this far-field flow angle trend is a result of viscous effects (possibly due to entrainment) or a result of wall effects needs to be investigated. Scans farther downstream, which would have been of interest, were not possible due to the limitations of the tunnel window size. Included in Fig. 6 are the near-field flow angles for $\alpha=6.2 \mathrm{deg}$. Noteworthy is the flow direction change at the shock wave, which is consistent with the shock orientation and strength at that location. These results demonstrate the degree to which these two optical techniques used in conjunction can quantify the inviscid flow.

\section{Turbulent Flow Properties}

As a first step in exploring the viscous flow region with the laser velocimeter, a streamwise station just downstream of the tfailing edge $(x / c=1.02)$ was surveyed. This station was sufficiently close to the trailing edge for the measured turbulent flow properties to represent the conditions at the trailing edge, yet sufficiently downstream that possible measurement difficulties associated with flare from the model's surface could be avoided. Figure 7 shows the mean velocity profiles obtained at this streamwise station for the three angles of attack selected for the study. The vertical distance is referenced to the trailing-edge location. Evident from this figure is the dramatic increase in boundary-layer thickness that occurs when the angle of attack is increased from 3.5 to $6.2 \mathrm{deg}$. (Recall that shock-induced separation has occurred in both cases.) Notice the upward displacement of the wake for the $6.2 \mathrm{deg}$ case. This rapid displacement of the wake was also apparent in the interferogram. Surprisingly, at this streamwise station wake closure has already occurred, even for the $\alpha=6.2 \mathrm{deg}$ case.

The corresponding turbulence intensities $\left\langle u^{\prime}\right\rangle$ and $\left\langle v^{\prime}\right\rangle$ and the velocity correlation $\overline{u^{\prime} v^{\prime}}$ plotted against $y / \delta$ are given in Figs. 8 and 9. The reader should note that each of the

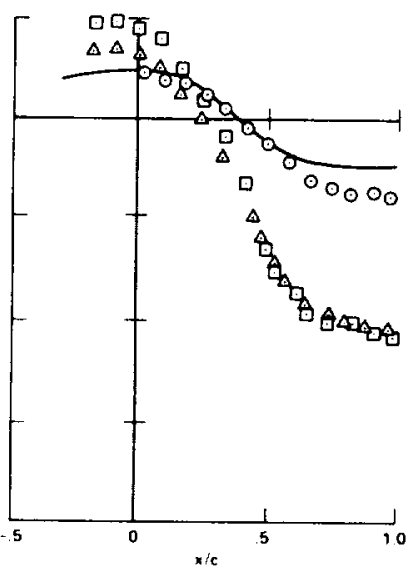

Fig. 6 Inviscid flow angles.

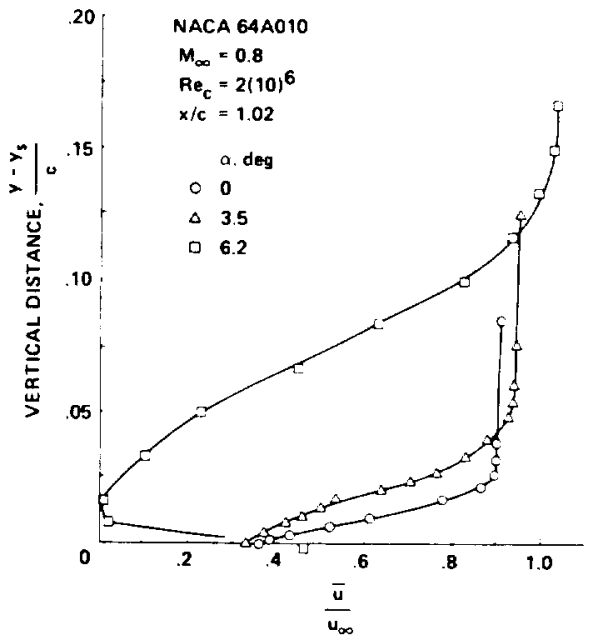

Fig. 7 Trailing-edge mean velocity profiles.

velocity correlation profiles (Fig. 9) is plotted on a different scale. From Fig. 8, it is seen that the fluctuation levels in both the streamwise and vertical direction increase with increasing angle of attack; however, the increases are greater for the streamwise fluctuations. The largest changes occur in the central portion of the layers where the increase in anistropy of the flow with angle of attack is apparent. For all three conditions, the maximum fluctuation levels occur away from the wall, as would be expected for a shear flow that has experienced an adverse pressure gradient.

The Reynolds shear stress for compressible flows is given by $-\bar{\rho} \overline{u^{\prime} v^{\prime}}$ (assuming that the triple correlation, $\overline{\rho^{\prime} u^{\prime} v^{\prime}}$, is negligible). However, in the transonic flow under investigation, the change in $\bar{\rho}$ across the viscous layer is quite small (e.g., $\bar{\rho}_{e} / \bar{\rho}_{w}=0.9$ for $M_{e}=0.8$ ) so the distribution of $-\overline{u^{\prime} v^{\prime} / u_{\infty}^{2}}$ is nearly the same as $-\tilde{\rho} u^{\prime} v^{\prime} / \rho_{w^{\prime}} u_{\infty}^{2}$. Thus, $-u^{\prime} v^{\prime} / u_{\infty}^{2}$ can, for practical purposes, be interpreted as the 
Fig. 8 Trailing-edge turbulence intensities.
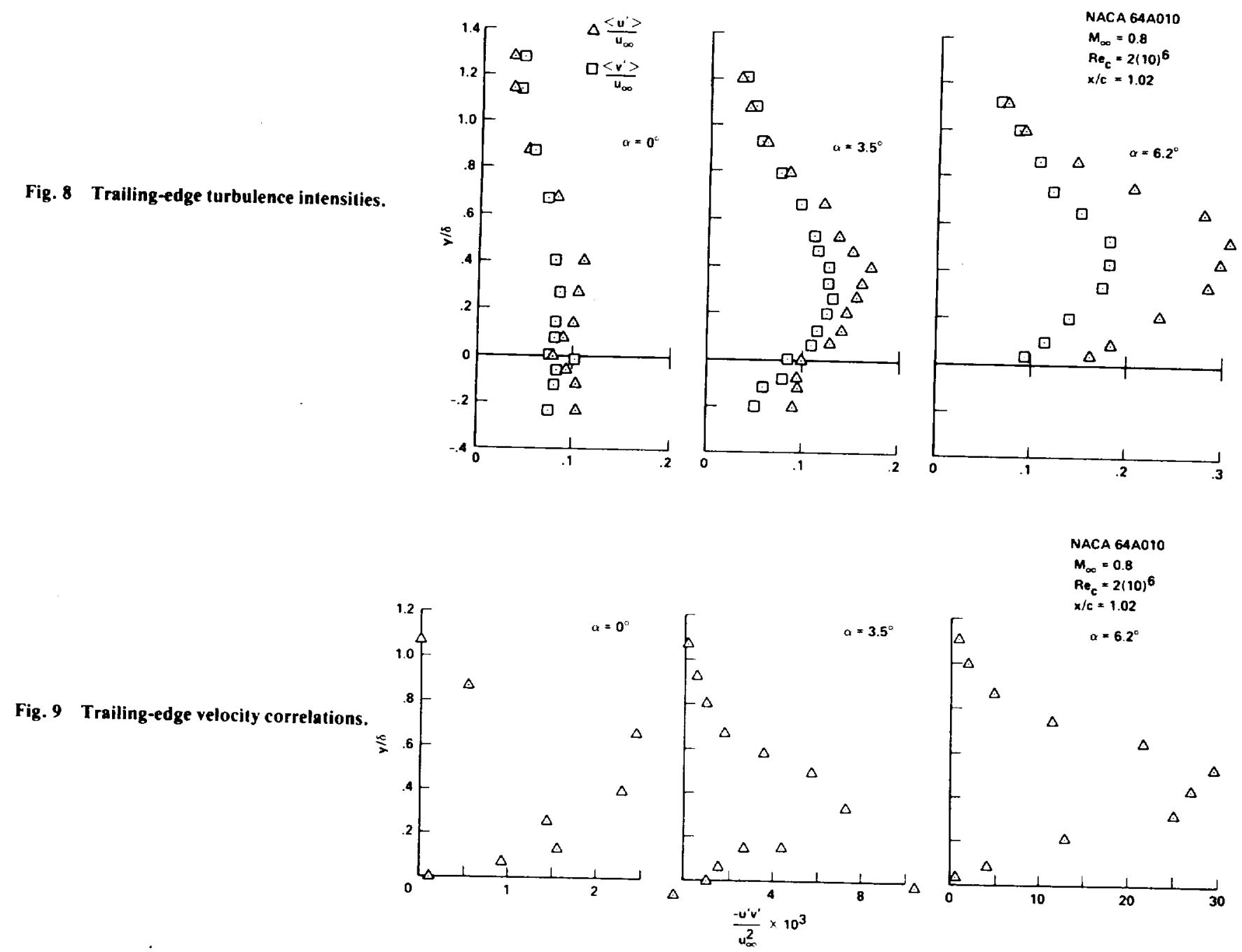

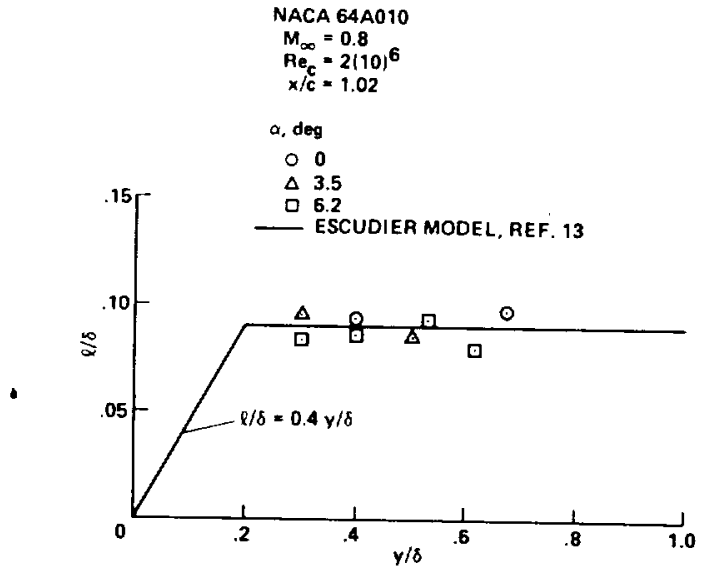

Fig. 10 Trailing-edge mixing lengths.

nondimensional Reynolds shear stress. In the region where the flow is most energetic, Fig. 9 shows that the velocity correlation and, accordingly, the Reynolds shear stress, increase dramatically (by over an order of magnitude between the 0 and $6.2 \mathrm{deg}$ cases), as do the turbulence intensities. This increase is very nearly equal to the change in turbulence kinetic energy, $q^{2}=\left(\left\langle u^{\prime}\right\rangle^{2}+\left\langle v^{\prime}\right\rangle^{2}+\left\langle w^{\prime}\right\rangle^{2}\right) / 2$, where $\left\langle w^{\prime}\right\rangle$ has been taken equal to $\left\langle v^{\prime}\right\rangle$.

From Figs. 7 and 9 it is seen that the point of maximum shear stress occurs where the strain rate $\partial \bar{u} / \partial y$ is the greatest. This would imply that an eddy viscosity description may be valid in describing the shear behavior. In Fig. 10, Prandtl's mixing length $\ell$ scaled to the boundary layer thickness

$$
\frac{\ell}{\delta}=\left(\overline{u^{\prime} v^{\prime}} \frac{1 / 2}{u_{\infty}^{2}}\right)^{1 / 2} / \frac{\partial\left(\bar{u} / u_{\infty}\right)}{\partial(y / \delta)}
$$

in the middle portion of the layers is compared to the model suggested by Escudier. ${ }^{13}$ Since accurate gradients are extremely difficult to obtain from experimental data, especially when the data are sparse, the determination of $\ell$ was only attempted in the region where the slope was nearly constant (i.e., where $\partial^{2} \bar{u} / \partial y^{2}$ was small) and thus, best defined. In fact, one slope was established for each of the three cases and the mixing lengths were calculated only for those points for which this constant slope appeared valid. The behavior of $\ell / \delta$ for $y / \delta<0.2$ is not discernible from these data due to the uncertainty of the strain rate in this region. It is astonishing that the calculated mixing lengths agree so well with the value of 0.09 , suggested by Escudier, in the middle portion of these layers. These three boundary layers have had entirely different flow histories, yet in the central portion of these layers equilibrium between the mean flow and the turbulent fluctuations appears to prevail at the trailing edge. The boundarylayer thickness just upstream of the shock wave is extremely thin. Thus, the trailing edge is many initial-boundary-layer thicknesses downstream of the interaction region, which may account for the unexpected equilibrium between the mean flow and the turbulent shear.

Measurements at several different streamwise locations were obtained for $\alpha=6.2 \mathrm{deg}$. This angle of attack was 

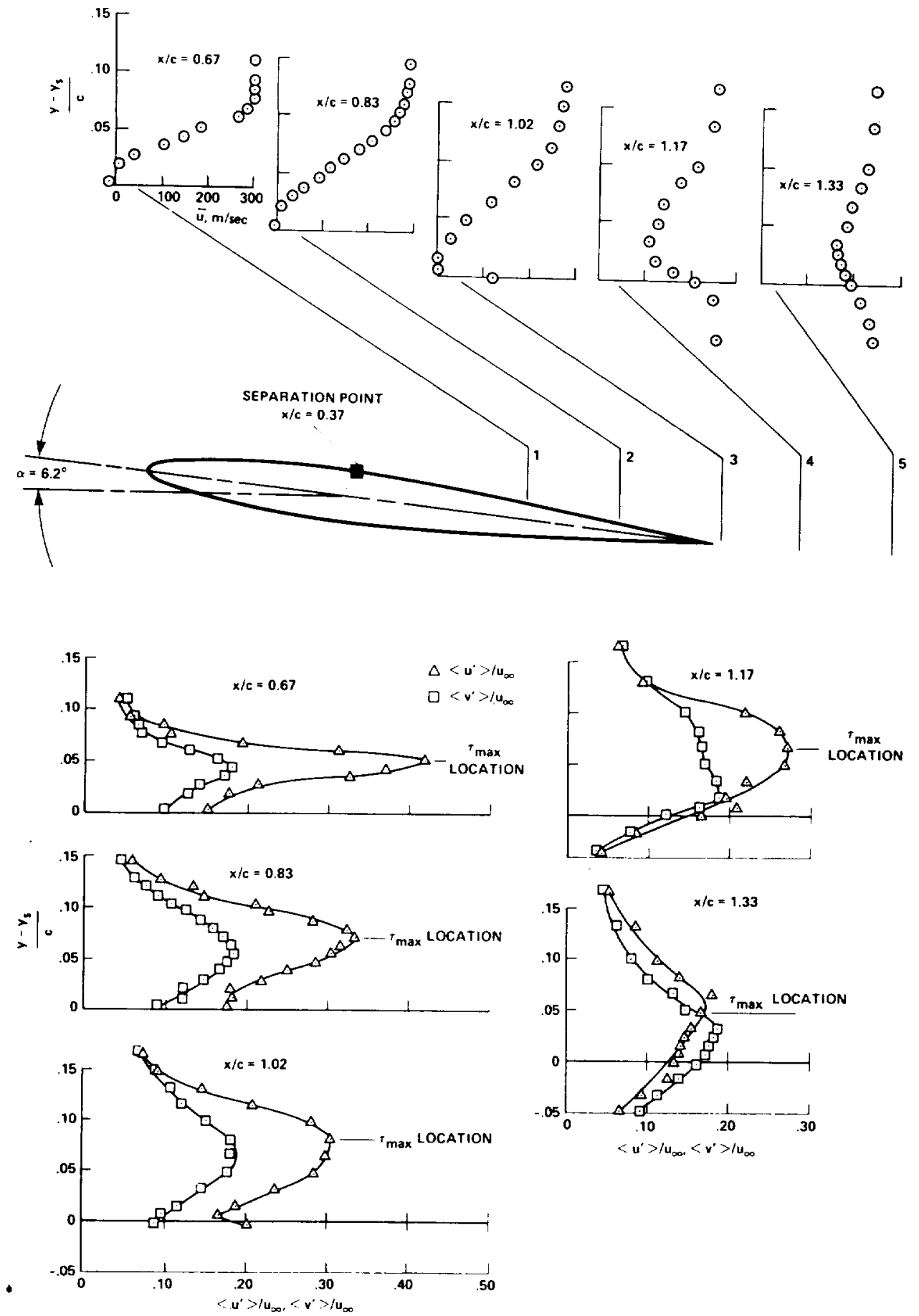

Fig. 12 Turbulence intensity distri.
butions: $\alpha=6.2$ deg, $M_{\infty}=0.8$, and $R e_{\mathrm{c}}=2 \times 10^{6}$.
Fig. 11 Mean velocily profiles: $\alpha=$ 6.2 deg; $M_{\infty}=0.8$, and $R e_{c}=$ $2 \times 10^{6}$. selected for studying the streamwise variation of the flow on the basis of: 1) the thick viscous layer present, which facilitated detailed probing, and 2) the inability of current numerical methods to predict even closely a flow with such a strong inviscid-viscous interaction. These data are presented in Figs. 11-13. For the measurement stations above the airfoil, the vertical distance is that from the surface, whereas in the wake it is referenced to the trailing-edge location. Along the airfoil, measurements were obtained within $0.5 \mathrm{~mm}$ of the surface. In Fig. 11, the profile plots are vertically displaced in accordance with the airfoil surface location. The furthermost upstream measurement station was $0.30 \mathrm{c}$ downstream of the separation point determined from oil flow visualization. Noteworthy from the mean velocity profiles are 1) the relatively small reversed velocities observed in the separated flow region, 2) the free-shear-layer shape to the profiles at the 

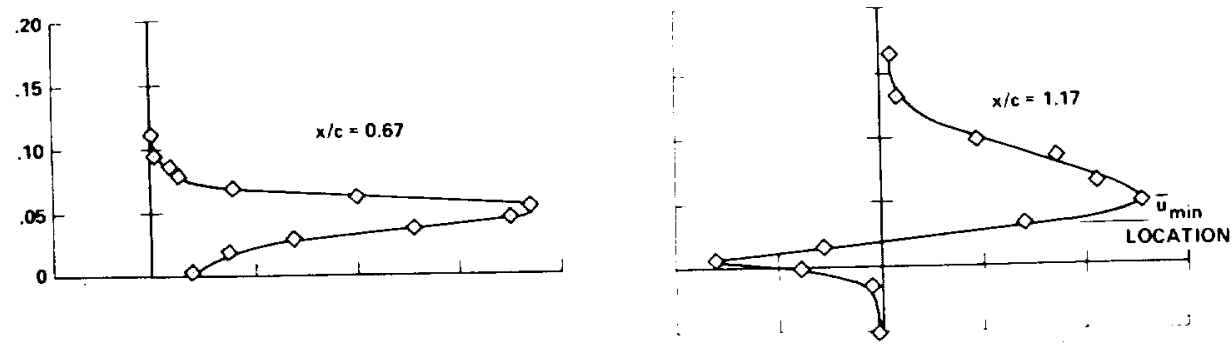

Fig. 13 Velocily correlation distributions: $\alpha=6.2$ deg, $M_{\infty}=0.8$, and $R e_{c}=2 \times 10^{6}$.
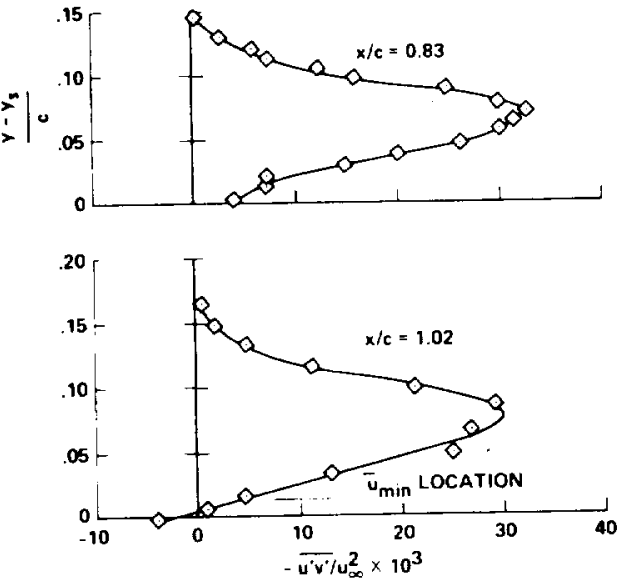

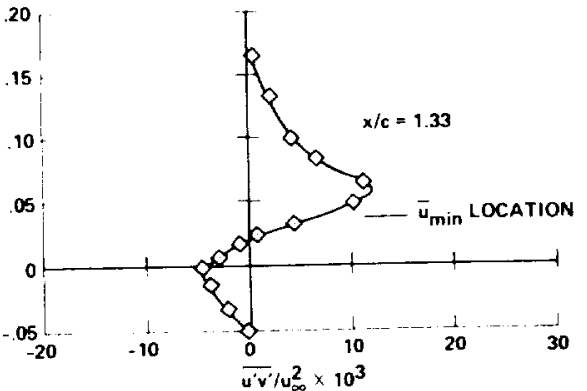

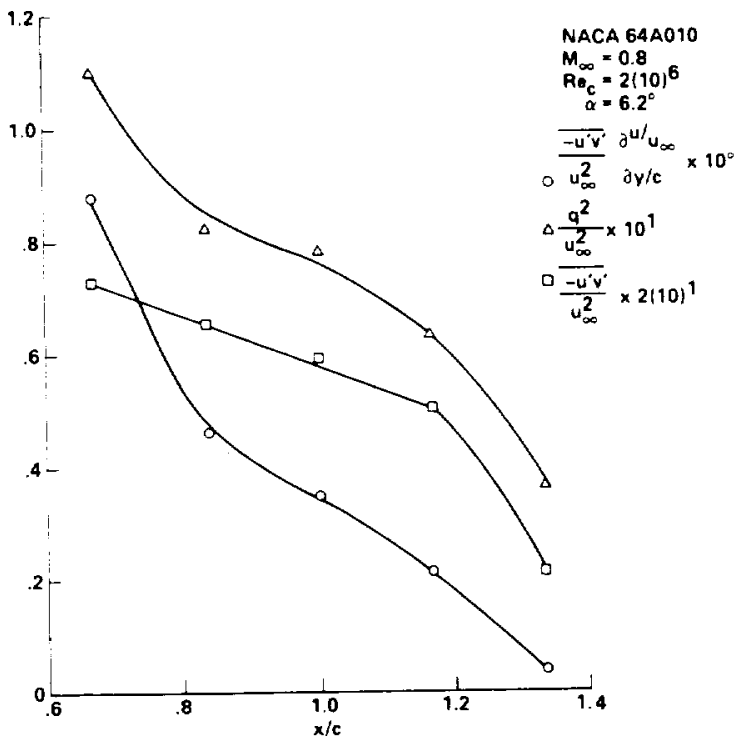

Fig. 14 Streamwise decay in production rale, turbulence kinetic energy, and shear.

Proceeding downstream, the streamwise fluctuations and, correspondingly, the turbulence kinetic energies are decaying, whereas the vertical fluctuations remain nearly constant. In the near wake (i.e., the last two downstream measurement stations), the character of the turbulence intensity distributions is seen to change rapidly; at the last station the flow has attained a nearly isotropic condition.

As seen from Fig. 13, the peak shear $\left(-\overline{u^{\prime} v^{\prime}} / u_{\infty}^{2}\right.$ is taken to represent $\left.\bar{\rho} \bar{u}^{\prime} v^{\prime} / \rho_{w} u_{\infty}^{2}\right)$ decreases with downstream distance as does the turbulence kinetic energy. At the last three stations, the presence of the lower surface boundary layer becomes evident from the sign change in the shear. Notice that the shear does not quite pass through zero where $\partial \bar{u} / \partial y=0$, (i.e., $\bar{u}_{\min }$ location) indicating nonequilibrium effects. The location of the peak shear $\tau_{\max }$ (i.e., the location where $-\overline{u^{\prime} v^{\prime}} / u_{\infty}^{2}$ is a maximum) was found in all cases to correspond to the location where $\left\langle u^{\prime}\right\rangle / u_{\infty}$ was also a maximum (see Fig. 12).

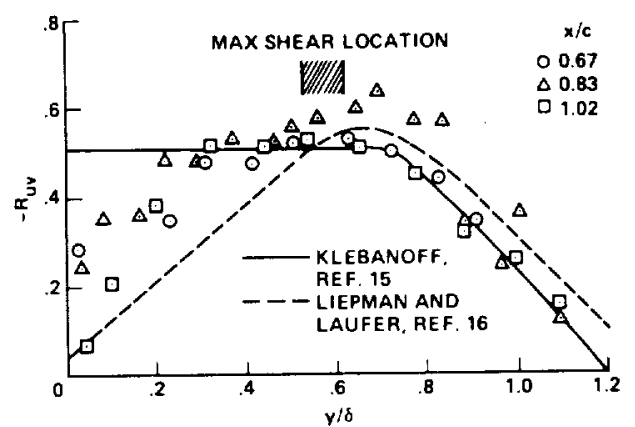

Fig. 15 Distribution of $u v$ correlation coefficient, $R_{u v}$.

A better illustration of the decay in the maximum shear stress and turbulence kinetic energy $\left(\left\langle w^{\prime}\right\rangle\right.$ taken equal to $\left.\left\langle v^{\prime}\right\rangle\right)$ with streamwise distance is presented in Fig. 14. Included in this figure is the quantity $u^{\prime} v^{\prime}(\partial \bar{u} / \partial y)$ scaled to $u_{\infty}$ and $c$. This term is the primary source term of turbulence kinetic energy for this flow. As seen from Fig. 14, the production rate of turbulence kinetic energy decreases rapidly with streamwise distance. This is due in a large part to the decrease in strain rate, $\partial \dot{u} / \partial y$, with increasing streamwise distance. With this rapid decrease in production rate, the corresponding rapid decay in turbulence kinetic energy is not surprising. These results, however, are contrary to those reported for a biconvex airfoil section at 0 deg angle of attack, where the shear and turbulence kinetic energy downstream of the shock wave and in the near wake were observed to increase with streamwise distance although the strain rate was rapidly decreasing. ${ }^{14}$ The observations of Ref. 14 may be a result of flow unsteadiness caused by having separation on both lower and upper surfaces.

The $u v$ correlation coefficients $R_{u v}$ for the three upstream stations are given in Fig. 15. In this figure the zero-pressure gradient boundary layer results of Klebanoff ${ }^{15}$ and the freeshear layer results of Leipmann and Laufer ${ }^{16}$ are also shown for comparison. As seen from Fig. 15, the present data could be represented quite well by a single curve. In the inner half of the boundary layer the correlation decreases. This behavior has also been observed for incompressible boundary layers subjected to an adverse pressure gradient. ${ }^{17}$ 


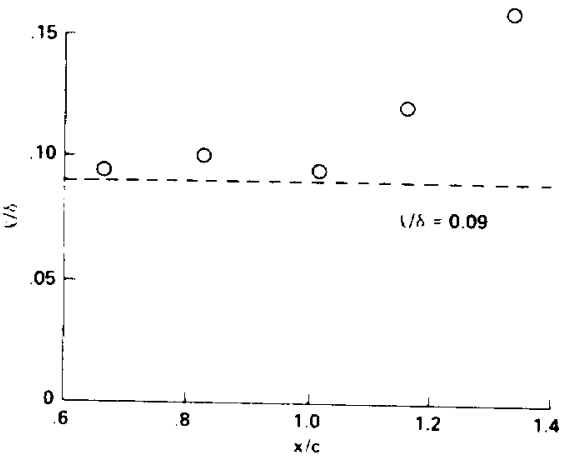

Fig. 16 Streamwise variation of mixing length at maximum shear.

The mixing lengths at the points of maximum shear (scaled to $\delta$ ) (this also corresponds to where the strain rate is a maximum) are presented in Fig. 16 as a function of distance along the airfoil. As shown earlier for the trailing-edge station, $\delta / \delta$ for the two upstream stations is well described by the value of 0.09 suggested by Escudier. In the wake, the mixing length scaled to $\delta$ (the distance from the $\bar{u}_{\min }$ location to the upper edge of the wake in this case) is increasing. This behavior in $\ell / \delta$ is reasonable considering that for an incompressible far-wake $i / \delta=0.18$ (Ref. 18). The mixing lengths in the lower part of the wake where $|\tau|$ attains another relative maximum are smaller as a result of the thinner lower surface boundary layer. These mixing lengths at $x / c=1.17$ and 1.33 are approximately one-third and one-half, respectively, those in the upper part of the wake, which indicates an even more rapid increase in mixing length with streamwise distance in this part of the wake.

The Escudier model has been shown to be reasonably accurate in relating the Reynolds shear stress to the strain rate $\partial \bar{u} / \partial y$ over the aft one-third of the airfoil's upper surface for an extremely strong interaction case and at the trailing edge for weaker interactions. Although measurements were obtained only at the trailing edge for the tw'o weaker interactions, the results for $\alpha=6.2$ deg strongly suggest the same would be true for these iwo cases. Yet, predictions for a $64 \mathrm{~A} 010$ airfoil section using this turbulence model have been found to be inaccurate for lifting cases. ${ }^{5}$ It appears that this mixing length model fails in the immediate vicinity of the shock wave. Extrapolation of the turbulence kinetic energy forward of the upstream-most measurement station in Fig. 14 suggests extremely high fluctuation levels at the stagnation point. An accurate accounting of the Reynolds normal stresses at and near the separation point may be what is needed to predict the initial perturbations to the flow. Once the initial perturbation to the boundary layer is accurately modeled, the present results suggest that a zero equation model such as Escudier's would be appropriate in the downstream regions if modifications were made to account for the increasing length scales in the nearwake region.

\section{Concluding Remarks}

Measurements of the flow behavior about a 64A010 airfoil section at transonic conditions have been presented. The results include Mach number contour plots and flow angles for the inviscid flow regions, and the turbulent flow properties of the upper surface viscous layer on the aft portion of the airfoil and of the near wake.

In view of the apparent inadequacies of current models for the turbulent Reynolds stresses, the turbulent flow measurements presented must be considered the most relevant results of the study. The present results show that equilibrium between the turbulent fluctuations and the local mean flow occurs much more rapidly in the central portions of the viscous layers than expected with $/ / \delta=0.9$ in this region. This condition was found to hold even at the furthermost upstream measurement station $(x / c=0.67)$ for $\alpha=6.2 \mathrm{deg}$. Although measurements were only obtained at the trailing edge for the two weaker interactions, the results for $\alpha=6.2 \mathrm{deg}$ strongly suggest the same would be true for these two cases. At $\alpha=6.2$ deg, measurements were obtained at several streamwise stations along the airfoil and in the near wake. These data reveal a rapid decay in the production rate of turbulence kinetic energy with streamwise distance. Associated with this rapid decay in production rate is a decay in the turbulence kinetic energy and Reynolds shear stress. In the near wake, the streamwise fluctuations decay more rapidly, with a near isotropic condition being established at $x / c=1.33$. As expected, the mixing length increases in the near wake as the flow establishes a wake character. The mean velocity measurements suggest a free-shear-layer type flow downstream of separation with very rapid closure of the wake near the trailing edge.

The results of this study suggest that it is at or very near the separation point that improved turbulence modeling is needed. Downstream of the separation point, a zero equation model would appear adequate. An accurate accounting of the Reynolds normal stresses at and near the separation point may be what is needed to predict the initial perturbations to the flow.

\section{References}

'Jameson, A., "Transonic Flow Calculations for Airfoils and Bodies of Revolution," Grumman Aerospace Corp., Bethpage, N.Y. Rept. 380-71-1, 1971.

2Bauer, F., Garabedian, P., and Korn, D., "A Theory of Supercritical W'ing Sections, with Computer Programs and Exam. ples," Lecture Notes in Economics and Mathematical Systems, Vol. 66, edited by M. Beckman and H.P. Kunzi, Springer-Verlag, N.Y. 1972

"Murman, E. M. and Cole, J. D., "Calculation of Plane Steady" Transonic Flow," AIAA Journal, Vol. 9, Jan. 1971, pp. 114-121.

${ }^{4}$ Deiwert, G. S., "Computation of Separated Transonic Turbulent Flows," AIAA Journal, Vol. 14, June 1976, pp. 735-740.

s Rose, W. C. and Seigner, A., "Calculation of Transonic Flow Over Supercritical Airfoil Sections," AIAA Paper 77-681, Albuquerque, N. Mex., 1977

${ }^{6}$ Braslow, A. L., Hicks, R. M., and Harris, R. V., Jr., "Use of Grit-Type Boundary-Layer-Transition Trips on Wind-Tunnel Models," NASA TN D-3579, 1966.

"McLaughlin, D. K., and Teiderman, W. G., "Biasing Corrections for Individual Realization of Laser Anemometer Measurements in Turbulent Flows," The Physics of of Fluids, Vol. 16, Dec. 1973, pp. 2082-2088.

${ }^{8}$ Bachalo, W. D., Modarress, D., and Johnson, D. A.. "Experiments on Transonic and Supersonic Turbulent Boundary Layer Separation," AIAA Paper 77-47, Los Angeles, Calif., 1977.

${ }^{9}$ Trolinger, J. D., "Conversion of Large Schlieren Systems to Holographic Visualization Systems," I5th National Aerospace Instrumentation Symposium, Las Vegas, Nev., May 1969.

${ }^{10}$ Trolinger, J. D., "Laser Instrumentation for Flow Field Diagnostics," AGARDograph 186, March 1974.

"Steinle, F. W., Jr. and Gross, A. R., "Pressure Dala from a $64 A 010$ Airfoil at Transonic Speeds in Heavy Gas Media of Ratio of Specific Heats from 167 to 112," NASA TM X-62,468, 1975.

${ }^{12}$ Stivers, L. S. Jr., "Effects of Subsonic Mach Number on the Forces and Pressure Distributions on Four 64A-Series Airfoil Sections at Angle of Altack," NACA TN 3162, 1954.

${ }_{13}^{13}$ Escudier, M. P., "The Distribution of the Mixing Length in Turbulent Flows Near Walls," Imperial College, London, Rept. TWF/TN/1, 1965 .

${ }^{14}$ Seegmiller, H. L., Marvin, J. G., and Levy, L. L. Jr., "Steady and Unsteady Transonic Flow," Paper 78-160, Huntsville, Ala.. 1978; also AIAA Journal, Vol. 16, Dec. 1978, pp. 1262-1270.

${ }^{15}$ Klebanoff, P. S., "Characteristics of Turbulence in a Boundary Layer with Zero Pressure Gradient," NACA Rept. 1247, 1955.

${ }^{16}$ Leipmann, H. W. and Laufer, J.. "Investigations of Free Turbulent Mixing," NACA TN-1257, 1947.

${ }^{17}$ Schubauer, G. B. and Klebanoff, P. S., "Investigation of Separation of the Turbulent Boundary Layer," NACA Rept. 1030, 1951.

${ }^{18}$ Schlichting, H., Boundary Layer Theory. McGraw-Hill, New York, Chap. 23, 1955 . 